\title{
artigo
}

Silva, E. C., Soares, P. F. C., Ferreira, J. D. L., Falcão, A. F., Leôncio, A. B. A., Braga, L. S.,

Percepção de mães acerca da violência obstétrica

\section{Percepção de mães acerca da violência obstétrica}

Mothers' perception about obstetric violence

Percepción de las madres sobre la violencia obstétrica

\section{RESUMO}

Objetivo: Compreender a percepção das mães acerca da violência obstétrica. Método: Pesquisa exploratória, descritiva, com abordagem quali-quantitativa. Realizada em três Unidades de Saúde da Família (USF) localizadas nos municípios de Pilar e Mari no estado da Paraíba, Brasil. A população do estudo foi constituída por todas as mães cadastradas nas USF's, porém, a amostra do estudo foi de 30 participantes. Na coleta de dados foram usados um questionário sócio demográfico e dados relacionados à violência obstétrica. Os resultados foram sintetizados no programa Statistic Package for Social Sciences (SPSS), versão 20.0. Resultados: 0 estudo apontou práticas rotineiras prejudiciais: teve acompanhante 6,35\%, manobra de kristeller 12, 23\%, episiotomia 16,53\%. Conclusão: A violência no campo da obstétrica precisa ser vencida a cada dia no Brasil e no mundo. Pesquisas como está, são importantes para ampliar o conhecimento e estimular boas práticas no parto.

DESCRITORES: Violência; Violência contra a mulher; Parto humanizado; Mães.

\section{ABSTRACT}

Objective: To understand the perception of mothers about obstetric violence. Methodo: Exploratory, descriptive research, with a quali-quantitative approach. Carried out in three Family Health Units (USF) maturing in the municipalities of Pilar and Mari in the state of Paraíba, Brazil. The study population was verified by all mothers registered in the USF's, however, the study sample consisted of 30 participants. A socio-demographic questionnaire and data related to obstetric violence were used in data collection. The results were synthesized in the Statistic Package for Social Sciences (SPSS), version 20.0. Results: The study pointed out harmful routine practices: there was a companion $6.35 \%$, kristeller maneuver $12,23 \%$, episiotomy $16.53 \%$. Conclusion: Violence in the field of obstetrics needs to be overcome every day in Brazil and worldwide. Research as it stands is important to expand knowledge and encourage good practices in childbirth.

DESCRIPTORS: Violence; Violence against women; Humanizing delivery; Molhes.

\section{RESUMEN}

Objetivo: Comprender la percepción de las madres sobre la violencia obstétrica. Método: Investigación exploratoria, descriptiva, con enfoque cuali-cuantitativo. Realizado en tres Unidades de Salud de la Familia (USF) en maduración en los municipios de Pilar y Mari en el estado de Paraíba, Brasil. La población de estudio fue verificada por todas las madres registradas en las USF, sin embargo, la muestra del estudio estuvo formada por 30 participantes. En la recopilación de datos se utilizó un cuestionario sociodemográfico y datos relacionados con la violencia obstétrica. Los resultados se sintetizaron en el paquete estadístico de ciencias sociales (SPSS), versión 20.0. Resultados: El estudio señaló prácticas rutinarias nocivas: hubo acompañante 6,35\%, maniobra de kristeller 12,23\%, episiotomía 16,53\%. Conclusión: La violencia en el campo de la obstetricia debe superarse todos los días en Brasil y en el mundo. La investigación tal como está es importante para ampliar los conocimientos y fomentar las buenas prácticas en el parto.

DESCRIPTORES: Violencia; La violencia contra las mujeres; Nacimiento humanizado; Madres.

RECEBIDO EM: 15/07/2021 APROVADO EM: 10/08/2021

\section{Edjane Carneiro da Silva}

Enfermeira.

ORCID:

\section{Perla Figueredo Carreiro Soares}

Mestre em Neurociência Cognitiva e Comportamento. Pós-graduada em enfermagem obstétrica. Chefe do Núcleo de Serviços Diagnósticos/SES/PB.

ORCID: 


\section{Josefa Danielma Lopes Ferreira}

Enfermeira. Mestra em enfermagem Programa de Pós-Graduação em Enfermagem da Universidade Federal da Paraíba (PPGENF/UFPB). Doutoranda em enfermagem pelo PGGENF/UFPB

ORCID:

\section{Aline Freire Falcão}

Enfermeira. Mestre em Educação pela Universidade Federal da Paraíba UFPB

ORCID:

\section{Alane Barreto de Almeida Leôncio}

Enfermeira. Mestre em Enfermagem pelo Programa de Pós-Graduação em Enfermagem da Universidade Federal da Paraíba (PPGENF/UFPB). Especialista em Saúde da Criança pelo Programa de Residência Multiprofissional do Estado da Paraíba.

ORCID:

\section{Luanna Silva Braga}

Enfermeira. Mestre em Enfermagem pelo Programa de Pós-Graduação em Enfermagem da Universidade Federal da Paraíba (PPGENF/UFPB).

ORCID:

\section{INTRODUÇÃO}

A $s$ mulheres são alvo de vários tipos de violência, além da violência intencional, os crimes contra a mulher são justificados por questões de níveis de desenvolvimento tanto econômico como social. A violência contra a mulher apresenta-se em diversas formas, dentre elas uma tem sido muito presente a violência obstétrica.

Existem diversos tipos de classificações de violência, e de acordo o Ministério da Saúde a violência contra a mulher se define por qualquer ação, discriminação, agressão seja essa pelo simples fato de ser a mulher a vítima e que cause possíveis danos como: constrangimento, sofrimento físico, sexual, moral, psicológico, social, político e até a morte.

As vítimas de agressōes ou de qualquer forma de violência contam com programas de apoio na maioria dos casos as queixas são de mulheres que são vítimas no próprio ambiente doméstico. A mulher vítima de violência também pode efetuar sua denúncia ou buscar orientação sobre como proteger-se do agressor no número 180 , que foi criado por força da Lei no 10.714 , de 13/08/03.

A Lei ordinária 11.340 foi promulgada em 07/08/2006, conhecida então, por "Lei Maria da Penha" e passou a prever penas mais duras para agressões contra mulheres,
As vítimas de agressões ou de qualquer forma de violência contam com programas de apoio na maioria dos casos as queixas são de mulheres que são vítimas no próprio ambiente doméstico aumentando os mecanismos de proteção das mesmas e alterando o previsto no Código Penal, permitindo flagrante criminal e a decretação da prisão preventiva do agressor. Outrossim, extinguiu as penas pecuniárias que estabeleciam cestas básicas ou multas.

A violência obstétrica, por não ter uma definição precisa, por vezes é relacionada exclusivamente com a experiência do parto. Contudo, é importante notar que ela abrange todos os outros domínios da saúde sexual e reprodutiva, como a anticoncepcional, o planejamento familiar, o aborto e a menopausa.

A Organização Mundial de Saúde (OMS), órgão fomentador e agregador de pesquisas em âmbito mundial, desaconselha que sejam feitas intervenções obstétricas desnecessárias por considerá-las um fator de risco tanto para a mãe como para o bebê, embora sejam, ainda, praticadas de forma rotineira no momento do parto, como é observável a partir da pesquisa, em que muitas mulheres vivenciam seus partos com estas intervenções, com ofensas, dor e humilhação.

A OMS declara, ainda, que toda mulher tem direito ao melhor padrão atingível de saúde, o qual inclui o direito a um cuidado de saúde digno e respeitoso. No mundo inteiro, muitas mulheres sofrem abusos, desrespeito e maus-tratos durante o parto nas instituições de saúde o que pode resultar em danos psicológicos e lesões que ficam 
para sempre no corpo da mulher.

Tal tratamento não apenas viola os direitos das mulheres ao cuidado respeitoso, mas também ameaça o direito à vida, à saúde, à integridade física e à não-discriminação. Esta declaração convoca maior ação, diálogo, pesquisa e mobilização sobre este importante tema de saúde pública e direitos humanos.

Estudos evidenciam que $25 \%$ das mulheres sofrem algum tipo de agressão durante a gestação, consultas pré-natais ou no parto. Estas agressões são praticadas por profissionais de saúde, tais como: repreensões, humilhações, gritos, ausência de oferta ou recusa nas práticas não farmacológicas de alívio da dor, realização de exames dolorosos e desnecessários, xingamentos grosseiros com características discriminatórias quanto à classe social ou cor da pele.

Agressões físicas ou verbais, procedimentos desnecessários ou sem consentimento da mulher, privação da presença do acompanhante escolhido pela mulher, não recebimento de métodos não farmacológicos de alívio para a dor e a separação de mãe e bebê saudável estão entre os abusos mais frequentes.

É direito da mulher ser informada de todas as etapas do trabalho de parto e parto, seja ele normal ou cesariana, além disso, a omissão de informações, prestação de informações em linguagem pouco acessível, desrespeito, desconsideração dos padrões e valores culturais das gestantes, e a negligência e recusa no atendimento, tanto para a gestante como para a mulher em situação de abortamento, também são considerados violência obstétrica. Dessa forma questionou-se: Como se dá a percepção das mães quanto a violência obstétrica? Tem-se por objetivo: Compreender a percepção das mães acerca da violência obstétrica.

\section{METODOLOGIA}

Pesquisa exploratória, descritiva, com abordagem quanti-qualitativa. Realizada três Unidades de Saúde da Família (USF) localizada nos municípios de Pilar e Mari no Estado da Paraíba, Brasil, no ano de 2016. A população foi constituída por to- das as mães cadastradas e acompanhadas nas USF's selecionadas. Porém, a amostra foi composta por 30 participantes, que foram selecionadas mediante a técnica não probabilística por conveniência. Para selecionar a amostra, foram elencados alguns critérios de inclusão: possuir idade superior a 18 anos, ter um filho e aceitar participar do estudo assinando o termo de consentimento livre e esclarecido (TCLE). Excluíram-se as mulheres com idade menor de 18 anos, que não tem filhos e que não aceitaram participar da pesquisa.

Foram usados um questionário socioeconômico e demográfico para caracterizar o perfil das mulheres e um questionário direcionado a violência obstétrica. Os dados da pesquisa foram analisados utilizando o tratamento estatístico a partir do programa Statistic Package for Social Sciences (SPSS), versão 20.0. A coleta de dados foi realizada no período de um mês, no ano de 2016, entre o primeiro e o semestre. A pesquisadora principal realizou a entrevista, $\mathrm{e}$ os participantes tiveram em média de 30 a 40 minutos para responder as questóes. A pesquisa seguiu os requisitos estabelecidos pela Resolução 466/12, sob o CAAE: 59903316100005176.

QUADRO 1 - Distribuição das respostas quanto as variáveis relacionadas ao tipo de parto segundo o questionário aplicado as participantes do estudo. Pilar e Mari, Paraiba, Brasil, 2016.

\begin{tabular}{|c|c|c|}
\hline VARIÁVEIS & PARTICIPANTES & PARTICIPANTES \\
\hline TIPO DE PARTO & N & $\%$ \\
\hline Normal & & \\
\hline Cesariana & 17 & 37 \\
\hline Fórceps & 11 & 3 \\
\hline Não respondeu & 01 & 3 \\
\hline Qual a posição do parto & 01 & 77 \\
\hline Deitada & & 23 \\
\hline Semideitada & 23 & \\
\hline TEVE LIBERDADE PARA & 07 & 7 \\
\hline ESCOLHER A POSIÇÃO & & 93 \\
\hline Sim & 02 & \\
\hline Não & 28 & \\
\hline Fonte: dados da pesquisa, 2016. & & \\
\hline
\end{tabular}


QUADRO 2 - Distribuição das respostas quanto as variáveis relacionadas ao acontecimento de alguma situação inesperada segundo o questionário aplicado as participantes do estudo. Pilar e Mari, Paraiba, Brasil, 2016.

\begin{tabular}{|c|c|c|}
\hline VARIÁVEIS & PARTICIPANTES & PARTICIPANTES \\
\hline & N & $\%$ \\
\hline $\begin{array}{c}\text { PASSOU POR ALGUM TIPO } \\
\text { DE SITUAÇÃO INESPERADA }\end{array}$ & & \\
\hline Sim & 16 & 53 \\
\hline Não & 14 & 47 \\
\hline Tipo de situação & & \\
\hline Foi mal atendida & 04 & 7 \\
\hline Não foi ouvida & 02 & 7 \\
\hline Agressão verbal & 02 & 3 \\
\hline Agressão física & 01 & 3 \\
\hline NÃo RESPONDEU & 01 & 36 \\
\hline Outros motivos & 06 & 93 \\
\hline Não & 28 & \\
\hline Fonte: dados da pesquisa, 2016. & & 7 \\
\hline
\end{tabular}

QUADRO 3 - Distribuição das respostas quanto as variáveis relacionadas as boas práticas de atenção ao parto, segundo o questionário aplicado as participantes do estudo. Pilar e Mari, Paraiba, Brasil, 2016.

\begin{tabular}{|c|c|c|}
\hline VARIÁVEIS & PARTICIPANTES & PARTICIPANTES \\
\hline & N & $\%$ \\
\hline FOI OFERECIDO ÁGUA OU ALIMENTO & & 23 \\
\hline Sim & 07 & 77 \\
\hline Não & 23 & \\
\hline FOI ORIENTADA A DEAMBULAR & & 47 \\
\hline Sim & 14 & 50 \\
\hline Não & 15 & 3 \\
\hline Não respondeu & 01 & 20 \\
\hline FOI OFERECIDO MÉTODOS & & 77 \\
\hline DE ALIVIO DA DOR & 06 & 3 \\
\hline Sim & 23 & 47 \\
\hline Não & 01 & 50 \\
\hline Não respondeu & & \\
\hline FOI REALIZADO ACESSO VENOSO & 14 & \\
\hline Sim & 15 & \\
\hline Não & & \\
\hline & & \\
\hline
\end{tabular}

relevância mal atendimento $(4 ; 25 \%)$.

A variável das mães que foi oferecida água ou alimentos $(7 ; 23 \%)$, visto que, nessas incluem também as mães que tiveram parto Cesária. Já na orientação quanto a deambulação a variável (14;47\%), como um todo deambular durante o trabalho de parto pode ajudar a mulher na diminuição de dores, bem como, a aceleração do parto. Bem como, na variável foi realizado acesso venoso o percentual $(14 ; 47 \%)$, as participantes que sofreram manobra de kristeller teve prevalência $(12 ; 40 \%)$, das mães que sofreram manobra de kristeller descarta-se a de parto cesariana.

A variável apresenta-se as mães que tiveram parto normal relataram o uso de ocitocina (11;37\%), no caso as que fizeram uso de ocitocina inclue-se as que tiveram filhos em parto normal, porém uma mulher que teve seu parto evoluído para uma cesariana declara ter feito uso de ocitocina, as participantes que foi realizado ocitocina sem consentimento prevaleceu (14;47\%).

De acordo com o resultado da questão das participantes que foram submetidas a episiotomia o resultado foi (16;53\%). Dessas mães as que consentiram a episiotomia teve a variável $(17 ; 91 \%)$, as que informaram que não consentiram o procedimento teve relevância $(16 ; 100 \%)$, neste contexto descartaram-se todas as mães que foram submetidas ao parto.

\section{DISCUSSÃO}

A mulher tem autonomia na escolha da via de parto, presumindo-se que a inclusão de suas preferências na decisão possa influenciar na hora do trabalho de parto. Um parto natural traz muitas vantagens, para ambos mãe e bebê, como resultados uma recuperação mais rápida, ausência de dor no período pós-parto, alta precoce, menor risco de infecção e de hemorragia. Por isto, a Organização Mundial da Saúde (OMS), preconiza que as cirurgias cesarianas deveriam corresponder a, no máximo, 15\% do total de partos e somente ser indicadas nos casos de risco para a mãe ou/o bebê.

De acordo com Câmara et al. a parturiente deve receber todas as informações 


\begin{tabular}{|c|c|c|}
\hline Não respondeu & 01 & 3 \\
\hline FOI REALIZADO MANOBRA DE KRISTELLER & & \\
\hline Sim & 12 & 40 \\
\hline Não & 17 & 57 \\
\hline Não respondeu & 01 & 3 \\
\hline
\end{tabular}

Fonte: dados da pesquisa, 2016

QUADRO 4 - Distribuição das respostas quanto as variáveis relacionadas ao uso de ocitocina, segundo o questionário aplicado as participantes do estudo. Pilar e Mari, Paraiba, Brasil, 2016.

\begin{tabular}{|c|c|c|}
\hline VARIÁVEIS & PARTICIPANTES & PARTICIPANTES \\
\hline & $N$ & $\%$ \\
\hline FOI REALIZADO OCITOCINA & & 37 \\
\hline Sim & 11 & 60 \\
\hline Não & 18 & 3 \\
\hline Não sabe & 01 & \\
\hline CONSENTIU A REALIZAÇÃo DE OCITOCINA & & 40 \\
\hline Sim & 07 & 3 \\
\hline Não & 12 & 3 \\
\hline Não respondeu & 01 & 36 \\
\hline Não respondeu & 01 & 93 \\
\hline Outros motivos & 06 & \\
\hline Não & 28 & \\
\hline Fonte: dados da pesquisa, 2016. & & \\
\hline
\end{tabular}

QUADRO 5 - Distribuição das respostas quanto as variáveis relacionadas a prática da episiotomia, segundo o questionário aplicado as participantes do estudo. Pilar e Mari, Paraiba, Brasil, 2016.

\begin{tabular}{|c|c|c|}
\hline VARIÁVEIS & PARTICIPANTES & PARTICIPANTES \\
\hline & $\mathrm{N}$ & $\%$ \\
\hline FOI REALIZADO EPISIOTOMIA & & \\
\hline Sim & 16 & 94 \\
\hline Não & 01 & \\
\hline CONSENTIU COM A EPISIOTOMIA & & 100 \\
\hline Não & 16 & \\
\hline Fonte: Elaborado pelo autor,2020. & & \\
\hline
\end{tabular}

necessárias para a prevenção e controle da ansiedade e do medo. Isso a tornará mais preparada para o fenômeno da parturição, podendo, inclusive, resultar na escolha mais adequada do tipo e posição do parto.

Atualmente a Organização Mundial de
Saúde, tem preconizado que a taxa de episiotomia não deve ultrapassar $10 \%$. Amorim et al. ressalta que, a episiotomia nunca seja realizada como um protocolo de não realização de episiotomia aliado a estratégias de proteção perineal, é importante lembrar que, como todo procedimento cirúrgico, a episiotomia só deveria ser realizada com o consentimento pós-informação da parturiente. O planejamento em relação a essa e outras intervenções também deve fazer parte do plano de parto.

Silva et al. aponta como consequências da realização da episiotomia a predisposição à mulher ao aumento de perda sanguínea, à infecção, disfunção sexual, a dispareunia, incontinência urinária e prolapso do colo do útero. Além disso, muitas vezes é realizado o "ponto do marido", para deixar a vagina mais apertada e preservar o prazer masculino, o que, por sua vez, para a mulher acarretará apenas dores na hora da relação sexual.

A Organização Mundial de Saúde, Visando a humanização, uso de técnicas não invasivas para alívio da dor como a deambulação durante o trabalho de parto é uma técnica utilizada com o propósito, além de outros, aliviar a dor sentida durante este período, embora nenhum autor explica como se dá esta, ofertar líquidos e alimentos, e liberdade de escolha da posição no parto. $\mathrm{O}$ uso da ocitocina sem consentimento, ainda é frequentemente realizada na assistência ao parto, podendo prejudicar a dinâmica do parto, como também, a oxigenação do bebê, comandos de puxo, mudança de ambiente, entre outros. Contudo, apesar do conhecimento, os profissionais continuam a realizá-la, apesar de jamais a registrarem em prontuário.

Segunde pesquisas afirma que a infusão de ocitocina desnecessária, determina maior percepção dolorosa, stress e medo na parturiente, podendo causar alguns efeitos colaterais para a mãe e o bebê. A parturiente pode ser acometida de taquissistolia, hipertonia e hiperestimulação uterina, podendo provocar inclusive a rotura uterina. A administração desse medicamento no pré-parto, ou seja, antes do segundo período do parto, é considerada uma prática prejudicial po- 
dendo trazer desvantagens e riscos, como a ruptura uterina e o sofrimento fetal agudo.

De acordo estas pesquisas a parturiente deve receber todas as informaçōes necessárias para a prevenção e controle da ansiedade e do medo. Isso a tornará mais preparada para o fenômeno da parturição, podendo, inclusive, resultar na escolha mais adequada do tipo e posição do parto, além do combate contínuo sob a violência obstétrica.

\section{CONCLUSÃO}

O tema demonstrou ser relevante em seus resultados, alcançando o objetivo inicial. Compreendeu-se que a violência obstétrica ainda ocasiona momentos difíceis na assistência ao parto e nascimento e que os profissionais precisam ampliar os seus conhecimentos sobre o assunto, retirando as práticas incabíveis do seu dia-a-dia. A violência obstétrica aparece de diversas formas durante o processo de parto, desde o consentimento de determinadas açōes junto a mulher, bem como, a ausência do acompanhamento, fato que é direito das mulheres. Dessa forma, novos estudos precisam ser disseminados, pois, o cuidado em saúde da mulher necessita de olhares e saberes atualizados, por isso, fez-se pertinente a propagação de pesquisas sobre o tema. A pesquisa apresentou como limitação científica, o fato de ter sido realizada no ano de 2016, e que em algum momento, pode não relatar pontos atuais vivenciados no ano de 2021, caracterizando como uma fragilidade no estudo.

\section{REFERÊNCIAS}

1. Andrade BP, Aggio CM. Violência obstétrica: a dor que cala: Simpósio Gênero e Políticas Públicas, Londrina, 2014.

2. Brasil. Ministério da Saúde. Manual técnico: pré-natal e puerpério. Brasillia; 2005.

3. Brasil. Lei $n^{\circ} 10.714$ de 13 de agosto de 2003. Autoriza o Poder Executivo a disponibilizar, em âmbito nacional, número telefônico destinado a atender denúncias 2 - de violência contra a mulher. 2003

4. Brasil. Parto e Nascimento domiciliar assistidos por Parteiras Tradicionais. 2006.

5. Belli L. Violência obstétrica: outra forma de violação dos direitos humanos. Revista Latina americana e Rede Caribenha de Bioética. ano 4, v. 1, n.7, jan.-jun. 2013.

6. Zanardo GLP, Calderón M., Nadal AHR., Habigzang, LF. Violência obstétrica no Brasil: uma revisão narrativa. Psicologia \& Sociedade, 29: e155043, 2017.

7. Organização Mundial de Saúde. Assistência ao parto normal: um guia prático. Saúde materna e neonatal. Unidade de maternidade segura. Saúde reprodutiva e da família. Genebra: OMS, 1996.

8. rganização Mundial de Saúde. Maternidade Segura. Assistência ao Parto Normal: um guia prático. Genebra, 2002. Rev. Rene. vol. 11, Número Especial, 2010. p. 92-98.

9. Fundação Perseu Abramo. Núcleo de Opinião Pública. Gravidez, filhos e violência institucional no parto. In: Mulheres brasileiras e gênero nos espaços público e privado: pesquisa de opinião pública. São Paulo: Fundação Perseu Abramo, 2010.

10. Brasil. Ministério da Saúde. Atenção humanizada ao abortamento: norma técnica / 2- Ministério da Saúde, 2. ed. - Brasília: Ministério da Saúde 2015.

11. Brasil. Parto, aborto e puerpério. 2001.

12. Câmara MFB, Medeiros M, Barbosa MA. Fatores sócio-culturais que influenciam a alta incidência de cesáreas e os vazios de assistência de enfermagem. Revista Eletrônica de Enfermagem. v 02, n. 01, 2000.
13. Amorim MMR de, Katz L. O papel da episiotomia na obstetrícia moderna. Revista Femina. v.36, p.47-54, 2008.

14. : Teorias, Estratégias e Implicações para Enfermeiros. Saúde das mulheres. NursWomens Health. 2012; v.16, p.210.

15. Silva NLS et al. Dispareunia, dor perineal e cicatrização após episiotomia. Revista de Enfermagem UERJ. v. 21, n. 2, p. 216220, 2012.

16. Leal MC. et al. Nascer no Brasil: inquérito nacional sobre parto e nascimento. Rio de Janeiro: ENSP/Fiocruz, 2012.

17. Carvalho VF de et al. Práticas prejudiciais ao parto: relato dos trabalhadores de saúde do sul do Brasil. Revista da Rede de Enfermagem do Nordeste-Rev Rene. v. 11, 2012.

18. LM Gomes de. et al. Violência obstétrica: perspectiva de puérperas atendidas em um hospital universitário no interior de Minas Gerais. Revista Saúde Coletiva Barueri, 2020. 